\title{
A higher degree of expression of DNA methyl transferase I in cervical cancer is associated with poor survival outcome
}

This article was published in the following Dove Press journal:

International Journal of Women's Health

8 June 2017

Number of times this article has been viewed

\author{
Chandrika J Piyathilake' \\ Suguna Badiga' \\ Samuel G Borak ${ }^{2}$ \\ Janaka Weragoda' \\ Sejong $\mathrm{Bae}^{3}$ \\ Roland Matthews ${ }^{4}$ \\ Walter C Bell ${ }^{2}$ \\ Edward E Partridge ${ }^{5}$ \\ 'Department of Nutrition Sciences, \\ 2Department of Pathology, \\ ${ }^{3}$ Department of Medicine, The \\ University of Alabama at Birmingham, \\ Birmingham, AL, ${ }^{4}$ Department \\ of Obstetrics and Gynecology, \\ Morehouse School of Medicine, \\ Atlanta, GA, ${ }^{5}$ Comprehensive Cancer \\ Center, The University of Alabama at \\ Birmingham, Birmingham, AL, USA
}

Correspondence: Chandrika J Piyathilake Department of Nutrition Sciences, The University of Alabama at Birmingham, Wallace Tumor Institute 420 D,

1824 6th Avenue South, Birmingham,

AL 35294-3300, USA

$\mathrm{Tel}+\mathrm{I} 2059755398$

Fax + I 2059347049

Email piyathic@uab.edu
Background: Even though novel therapies based on aberrant DNA methylation could be of particular importance for the treatment of cervical cancer (CC) because the oncoproteins E6/E7 of high-risk human papillomaviruses, the causative agents for developing $\mathrm{CC}$, have the capacity to bind and upregulate DNA methyltransferases (DNMTs), to our knowledge, no previous studies have evaluated the expression of this enzyme in CC in relation to survival outcomes. The purpose of the study was to evaluate the expression of DNMT1 in CC and its association with survival outcomes.

Methods: The study population consisted of 76 women treated for primary $\mathrm{CC}$ and followed up by the University of Alabama at Birmingham (UAB) cancer registry. The expression of DNMT1 was examined using immunohistochemistry, and the degree of expression of DNMT1 was expressed as a percentage of cells positive for DNMT1 and its intensity. Cox proportional hazards model was used to assess the relationship between the degree of expression of DNMT1 and overall survival after adjusting for relevant covariates.

Results: The expression of DNMT1 was significantly higher in CC cells compared to that in the normal cervical epithelium. A higher percentage of cells positive for DNMT1 and a higher intensity score for DNMT1 were significantly associated with poor survival outcome (hazard ratio $[\mathrm{HR}]=4.3, P=0.03$ and $\mathrm{HR}=4.9, P=0.02$, respectively).

Conclusion: Our findings suggested that the degree of expression of DNMT1 could be considered as a target in the epigenetic treatment of CC. Replication of our results in other study populations with CC could create the opportunity of using DNMT inhibitors to treat CC.

Keywords: DNMT1, cervical cancer, survival outcome

\section{Introduction}

Cervical cancer (CC) is the third most commonly diagnosed cancer and the fourth leading cause of cancer death in women worldwide. ${ }^{1}$ The American Cancer Society estimated that in the United States, 12,990 new cases were diagnosed and 4,120 died from this cancer in $2016 .^{2}$ The addition of platinum-based chemotherapy to adjuvant radiotherapy (chemoradiation) $)^{3}$ has improved the survival of advanced-stage $\mathrm{CC}$ patients over radiotherapy alone, but the 5-year relative survival remains $57 \%$ and $16 \%$ for regional and distant disease, respectively. ${ }^{4}$ Since $\mathrm{CC}$ affects relatively young women in their prime of life, especially in developing countries, it is an important cause of lost life-years affecting not only the woman but also the well-being of the entire family as women play a major role in driving a successful family environment. This observation indicates the need for novel and tailored treatments to improve the outcomes in this disease, especially in developing countries where $\mathrm{CC}$ is diagnosed at advanced stages due to lack of screening programs. 
Novel therapies based on aberrant DNA methylation could be of particular importance for the management of CC because the oncoproteins E6/E7 of high-risk human papillomaviruses (HR-HPVs), the causative agents for developing $\mathrm{CC}$, have the capacity to bind and upregulate DNA methyltransferases (DNMTs), the enzymes responsible for the methylation of CpGs in mammals. Despite this plausibility for higher expression of DNMTs in CC and that aberrant DNA methylation associated with DNMT1 plays pivotal roles in the neoplastic progression via $\mathrm{CpG}$ island hypermethylation associated with gene silencing, ${ }^{5,6}$ to our knowledge, no previous studies have evaluated the expression of this enzyme in CC in relation to survival outcomes. Documentation of the degree of expression of DNMT1 and its association with $\mathrm{CC}$ outcomes is important since it may create the opportunity of using DNMT inhibitors (DNMTis) to treat CC. This is particularly important because a growing body of research has shown that DNMTis are also capable of upregulating immune signaling in epithelial cancer cells through demethylation of endogenous retroviruses and cancer testis antigens. In addition, clinical trials are currently testing the efficacy of combining epigenetic therapy and immunotherapy to treat certain cancers, but not CC. ${ }^{7}$

Based on this background, the purpose of this study was to evaluate the expression of DNMT1 in CC and its association with survival outcomes.

\section{Methods}

\section{Study population}

The study population consisted of 76 women (20 African American [AA] and 56 Caucasian American [CA]) who were treated for primary CC and followed up by the University of Alabama at Birmingham (UAB) cancer registry. Paraffinembedded specimens of cancer tissues were available for all women. Women were diagnosed with either cervical adenocarcinoma (AC; $\mathrm{n}=35$ ) or squamous cell carcinoma (SCC; $n=41$ ). Information on age, race, mode of treatment, stage and vital status was provided by the cancer registry. The patients were not given chemotherapy or radiation prior to surgery and were subsequently treated with such therapies. The staging of CCs was based on the American Joint Committee on Cancer TNM staging system. The study was approved by the institutional review board of UAB with a waiver of informed consent as we did not have direct contacts with our study participants. In order to meet patient data confidentiality, all specimens used in the study were stripped of identifiers and only coded identifiers were used throughout the study.

\section{Immunohistochemical assay for DNMTI}

Immunohistochemical staining and its assessment for DNMT1 were performed following a previously published protocol using a polyclonal antibody specific for DNMT1 (H-300, sc-20701). ${ }^{8}$ Briefly, the slides containing $4 \mu \mathrm{m}$-thick paraffin tissue sections were deparaffinized in xylene, placed in $0.01 \mathrm{M}$ citric acid, $\mathrm{pH} 6.0$, and boiled in a pressure cooker set at full power for 10 minutes. After antigen retrieval, the sections were cooled, rinsed in deionized $\mathrm{H}_{2} \mathrm{O}\left(\mathrm{dH}_{2} \mathrm{O}\right)$ and placed in $3.5 \mathrm{~N} \mathrm{HCl}$ for 15 minutes at room temperature to open the DNA. The sections were rinsed with $\mathrm{dH}_{2} \mathrm{O}$ and treated with $3.0 \% \mathrm{H}_{2} \mathrm{O}_{2}$ for 5 minutes to quench endogenous peroxidase activity. Sections were incubated with preimmune goat serum (3\%) for 20 minutes at room temperature to suppress nonspecific staining and then subsequently incubated with DNMT1 antibody (1:100 concentration) for 1 hour at room temperature. After washing thoroughly with Tris-buffered saline, the primary antibody was detected using a multispecies system (Signet Laboratories, Inc., Dedham, MA, USA). The antibody-antigen complex was visualized using a 3,3-diaminobenzidine substrate kit according to the manufacturer's instructions (Biogenex, Inc., San Ramon, CA, USA) and lightly counterstained with hematoxylin. Slides were sequentially dipped in 70\%, 95\% and $100 \%$ ethanol ( 3 minutes each) and were then allowed to dry slightly. Finally, slides were dipped in xylene three times (3 minutes each) after which they were protected with cover slips.

\section{Assessment of immunostaining}

Immunostaining for DNMT1 was localized mainly in the nuclei of cells. Nuclear staining in cells with or without cytoplasmic staining was interpreted as a positive reaction. The degree of expression of DNMT1 in the nuclei was recorded independently by two observers (WCB and SGB) as a percentage of cells positive for DNMT1 based on a visual assessment of the intensity of brown reaction product within the cell nucleus on a scale of 0 (no staining) to $4+$ (intense staining). All evaluations were done separately in the basal, bottom-half and top-half layers of the normal cervical epithelium. This approach is time consuming but allows obtaining a more accurate estimate on the percentage of cells positive for DNMT1 in the normal cervical epithelium. One reading was given for cancerous lesions. The degree of expression of DNMT1 was reported as the total percentage of cells positive at any intensity $(1-4+)$ and as an intensity score that was derived by multiplying the percentage of cells positive for DNMT1 at each intensity 
score by the appropriate intensity score. Except for CC, the percentage of cells positive for DNMT1 or the intensity score in the basal, bottom-half and top-half layers of the normal epithelium was averaged to obtain the average degree of DNMT1 expression. The readings from the two observers were averaged for each cell or lesion type (normal or CC), and thus, the degree of DNMT1 expression reported is the average of the two observers.

\section{Data analysis}

The correlation between the percentages of cells positive for DNMT1 and its intensity score was tested using Spearman's rho $(r)$ correlation coefficient. The differences in the median percentage of cells positive for DNMT1 and the median intensity score for DNMT1 between the normal cells and $\mathrm{CC}$ cells found in the same specimen of AC and SCC were determined using the median test. We tested the differences in the characteristics of the women (age, race, stage of cancer, mode of treatment, histological subtype of cancer, survival in months and vital status) by median percentage of cells positive for DNMT1 (median $=77.5 \%$ ) and the median intensity score for DNMT1 (median =0.9625) using Pearson's chi square test for categorical variables and median test for continuous variables. The median percentage of cells positive for DNMT1 or the median intensity score was based on the entire sample, AC and SCC combined $(n=76)$. We assessed the relationship between the median percentage of cancer cells positive for DNMT1 and the median intensity score for DNMT1 in those cells and overall survival after adjusting for covariates, namely, age, race (AA vs CA), histological subtypes of CC (AC vs SCC), stage of the cancer (1 and 2 vs 3 and 4) and the mode of treatment (surgery alone vs surgery along with radiation therapy or chemotherapy or both). In addition, we also determined whether overall survival differed between ACs expressing higher degree of DNMT1 compared to SCCs expressing a similar degree of DNMT1. The overall survival was defined as the time elapsed between the date of diagnosis and death or the date of last contact. Survival curve was estimated by the Kaplan-Meier method, and a multivariate analysis was performed using Cox's proportional hazard regression model. Because of the small sample size of the study, we did not stratify the data by race or subtype of the $\mathrm{CC}$.

\section{Results}

The study population consisted of $46 \%$ and $54 \%$ of women diagnosed with cervical AC and SCC, respectively. Spearman's rho $(r)$ correlation coefficient between the percentages of cells positive for DNMT1 and its intensity score was 0.95 for both normal and cancer cells $(P<0.0001)$. Figure 1 shows the categorical scatter plot of percentage of cells positive for DNMT1 (A) and intensity score for DNMT1 (B) in normal and cancer cells in both AC and SCC specimens combined and separately. The median percentage of cancer cells
A

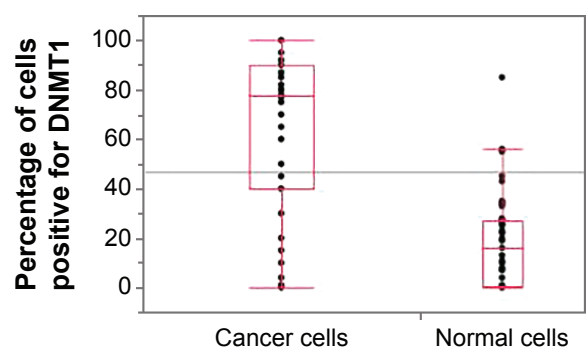

D

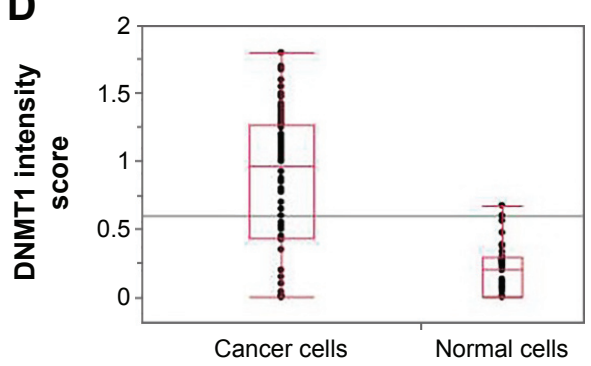

B

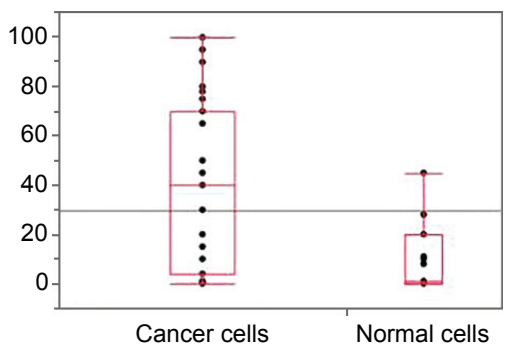

E

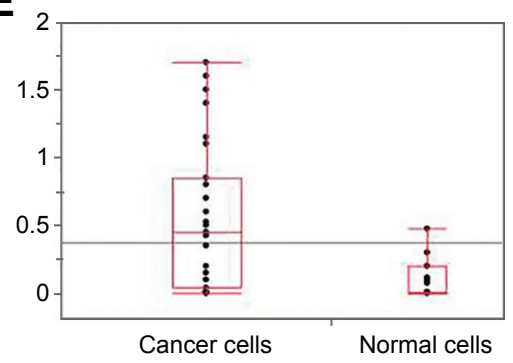

C

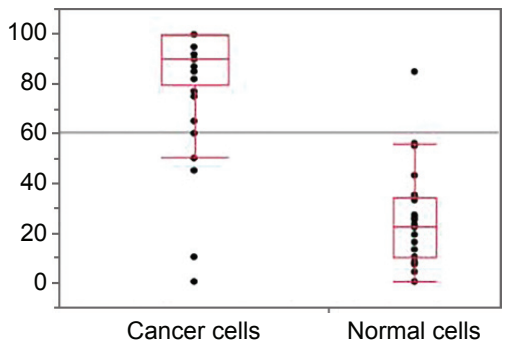

F

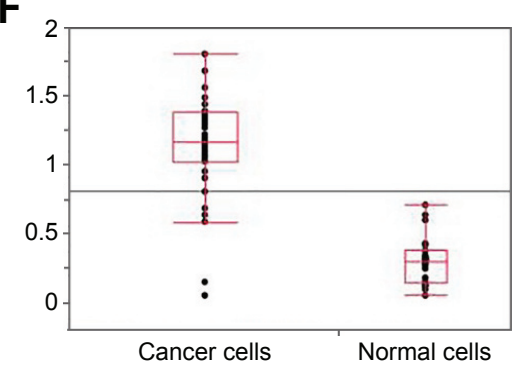

Figure I Scatter plots of the percentages of cancer cells and normal cells positive for DNMTI among (A) AC and SCC combined, (B) AC and (C) SCC, and scatter plots of the DNMTI intensity score of cancer cells and normal cells among (D) AC and SCC combined, (E) AC and (F) SCC.

Abbreviations: DNMT, DNA methyltransferase; AC, adenocarcinoma; SCC, squamous cell carcinoma. 
positive for DNMT1 (77.5\%) was significantly higher than the median percentage of normal cells positive for DNMT1 $(16 \%)(P<0.0001)$ (Figure 1A). Similarly, the median intensity score for DNMT1 in cancer cells (1.0) was significantly higher than the median intensity score for DNMT1 in normal cells $(0.2)(P<0.0001)$ (Figure 1D). When we analyzed the difference between the expression of DNMT1 in normal and cancer cells by histological subtypes of $\mathrm{CC}$, we observed a similar pattern and the differences were statistically significant (percentage of cells positive for DNMT1, normal vs cancer for AC 1.0 vs 40 [ $P=0.0096]$; SCC 22.5 vs 90.0 $[P<0.0001]$ (Figure $1 \mathrm{~B}$ and $\mathrm{C}$ respectively); intensity score for DNMT1, normal vs cancer for AC 0.0 vs $0.5[P=0.0096]$; SCC 0.3 vs $1.1[P<0.0001]$ [Figure $1 \mathrm{E}$ and $\mathrm{F}$, respectively]). However, we observed that the percentage of cells positive for DNMT1 and the intensity score for DNMT1 in AC specimens were lower compared to SCC specimens in both normal (percentage of cells positive for DNMT1 AC vs SCC, $1 \%$ vs $22.5 \%[P=0.0088]$ and intensity score for DNMT1 AC vs SCC, 0 vs $0.3[P=0.0018]$ ) and cancer cells (percentage of cells positive for DNMT1 AC vs SCC, $40 \%$ vs $90 \%$ $[P<0.0001]$ and intensity score for DNMT1 AC vs SCC, 0.5 vs $1.1[P<0.0001])$. Figure 2 illustrates the expression of DNMT1 in normal and cancer cells of AC and SCC.

The characteristics of the study population by the median percentage of cells positive for DNMT1 and DNMT1 intensity score in cancer cells are shown in Table 1 . We observed that CA women (87\%) were significantly more likely to have a higher percentage of cancer cells positive for DNMT1 and a higher intensity score for DNMT1 compared to AA women (13\%) $(P=0.0092)$. We did not observe a significant difference in other characteristics of the population, namely, age, stage of diagnosis, mode of treatment, survival in months and vital status by the median percentage of cancer cells positive for DNMT1 or the median intensity score for DNMT1 in cancer cells. However, we observed that $82 \%$ of SCC specimens had higher than median percentage of cancer cells positive for DNMT1 compared to $18 \%$ of AC specimens. Similarly, $84 \%$ of SCC specimens had higher than median intensity score for DNMT1 compared to $16 \%$ of AC specimens.

Figure 3 shows the survival curve for the association between median percentage of cells positive for DNMT1 and median intensity score for DNMT1 and overall survival from CC adjusted for age, race, histological subtypes of CC, stage of cancer and mode of treatment. Those with a $>77.5 \%$ of cells positive for DNMT1 were 4.3 times more likely to die earlier compared to women with lower expression of this marker (hazard ratio $[\mathrm{HR}]=4.3 ; 95 \%$ confidence interval [95\% CI] $=1.1-19.7 ; P=0.03$ ) (Figure 3A). We observed a similar association between the intensity score of DNMT1 and survival from $\mathrm{CC}$ after adjusting for the abovementioned variables. Those with a higher than 0.9625 intensity score of DNMT1 were 4.9 times more likely to die earlier compared to women with lower expression of this marker ( $\mathrm{HR}=4.9 ; 95 \% \mathrm{CI}=1.2-23.3 ; P=0.02$ ) (Figure 3B). We also observed that ACs expressing higher degree of DNMT1 were six times more likely to have poor survival compared
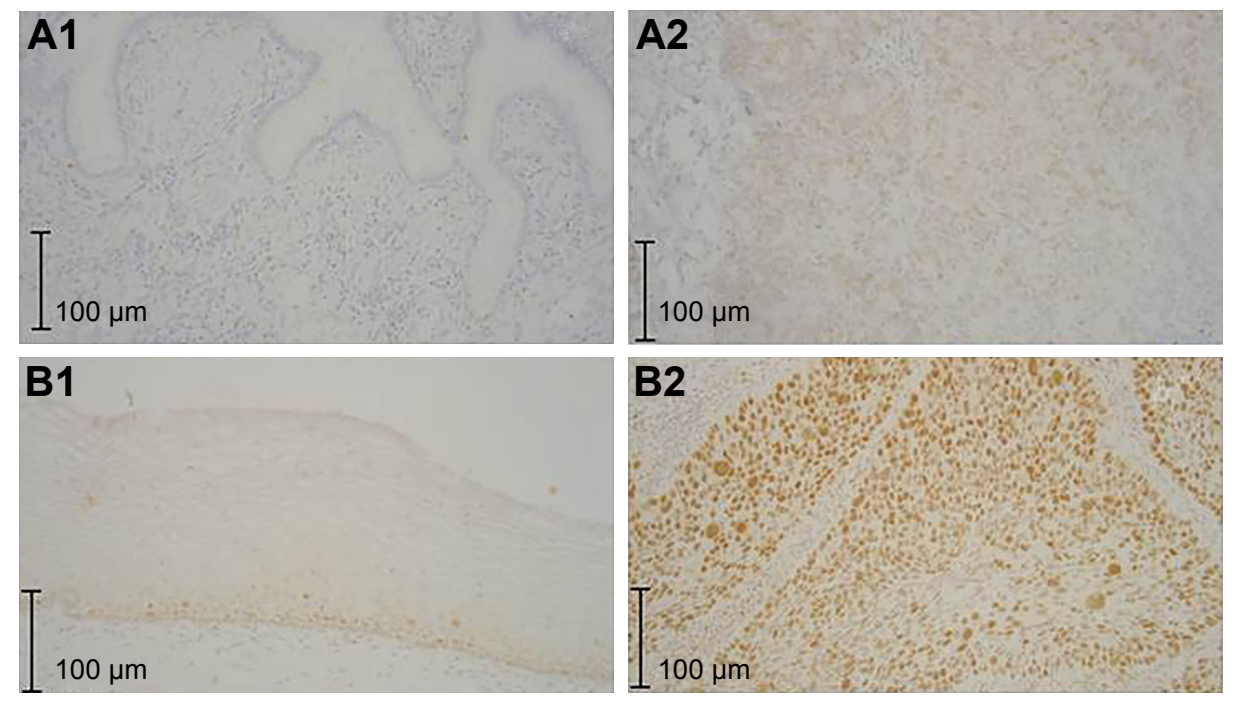

Figure 2 Expression of DNMTI in (AI) normal endocervical glands, essentially no staining, (A2) AC, (B I) normal squamous epithelium, staining largely limited to basal and parabasal regions and (B2) SCC.

Abbreviations: DNMT, DNA methyltransferase; AC, adenocarcinoma; SCC, squamous cell carcinoma. 
Table I Characteristics of the population by expression of DNMTI

\begin{tabular}{|c|c|c|c|c|c|c|}
\hline \multirow[t]{2}{*}{ Characteristics } & \multicolumn{2}{|c|}{$\begin{array}{l}\text { Cancer cells positive for } \\
\text { DNMTI expression }\end{array}$} & \multirow[t]{2}{*}{$P$-value } & \multicolumn{2}{|c|}{$\begin{array}{l}\text { Intensity score of the } \\
\text { cancer cells for DNMTI }\end{array}$} & \multirow[t]{2}{*}{$P$-value } \\
\hline & $\leq \mathbf{7 7 . 5} \%$ & $>77.5 \%$ & & $\leq 0.9625$ & $>0.9625$ & \\
\hline Age, median (years) & 44.5 & 43.5 & 0.4026 & 43.5 & 44.5 & 0.8173 \\
\hline \multicolumn{7}{|l|}{ Race, n (\%) } \\
\hline AA & 15 (39\%) & $5(13 \%)$ & 0.0092 & 15 (39\%) & $5(13 \%)$ & 0.0092 \\
\hline $\mathrm{CA}$ & $23(41 \%)$ & $33(87 \%)$ & & $23(61 \%)$ & $33(87 \%)$ & \\
\hline \multicolumn{7}{|l|}{ Stage of diagnosis, n (\%) } \\
\hline $\mathrm{I}$ and 2 & $30(79 \%)$ & $30(79 \%)$ & 1.000 & 30 (79\%) & 30 (79\%) & 1.000 \\
\hline 3 and 4 & $8(21 \%)$ & $8(21 \%)$ & & $8(21 \%)$ & $8(21 \%)$ & \\
\hline \multicolumn{7}{|c|}{ Mode of treatment, $\mathrm{n}(\%)$} \\
\hline Surgery only & $20(53 \%)$ & $25(66 \%)$ & 0.2424 & $20(53 \%)$ & $25(66 \%)$ & 0.2424 \\
\hline $\begin{array}{l}\text { Surgery along with } \\
\text { radiation or chemot }\end{array}$ & $18(47 \%)$ & $13(34 \%)$ & & $18(47 \%)$ & $13(34 \%)$ & \\
\hline \multicolumn{7}{|c|}{$\begin{array}{l}\text { Histological subtype of cervical } \\
\text { cancer, } n(\%)\end{array}$} \\
\hline $\mathrm{AC}$ & $28(74 \%)$ & 7 (I8\%) & $<0.0001$ & $29(76 \%)$ & $6(16 \%)$ & $<0.0001$ \\
\hline SCC & $10(26 \%)$ & $31(82 \%)$ & & $9(24 \%)$ & 32 (84\%) & \\
\hline Survival in months & 53.21 & 58.12 & 0.6467 & 55.82 & 56.64 & 1.000 \\
\hline \multicolumn{7}{|l|}{ Vital status, n (\%) } \\
\hline Dead & $10(26 \%)$ & $9(24 \%)$ & 0.7911 & $10(29 \%)$ & $9(21 \%)$ & 0.4242 \\
\hline Alive & $28(74 \%)$ & $29(76 \%)$ & & 24 (7।\%) & $33(79 \%)$ & \\
\hline
\end{tabular}

Abbreviations: DNMT, DNA methyltransferase; AA, African American; CA, Caucasian American; AC, adenocarcinoma; SCC, squamous cell carcinoma.

to SCCs expressing similar degree of DNMT1 $(P=0.0177)$ after adjusting for relevant covariates.

\section{Discussion}

Even though downward trends in CC have been observed in high-income countries, no clear changes, or an increase in risk, is found in low-income countries. ${ }^{9}$ Worldwide ${ }^{10,11}$ and in the United States, ${ }^{12} \mathrm{SCC}$ is the predominant histological type accounting for approximately three-quarters of all CCs. However, a rising incidence of cervical AC relative to SCC has been reported in the United States and in Europe in recent decades. ${ }^{13,14}$ Even though infections with HR-HPVs is recognized as the main causative agent for developing $\mathrm{CC}, \mathrm{SCC}$ is more likely to be positive (87\%) compared to $\mathrm{AC}(62 \%) .{ }^{15}$ Based on this difference in the prevalence of HR-HPVs, it is plausible that the degree of expression of E6/E7 could be lower in AC compared to SCC, and therefore, the capacity to bind and upregulate DNMT1 and the resulting expression of this marker as we have observed could also be lower in AC compared to SCC. A higher expression of DNMT1 in cancer tissues compared to normal tissues may also be explained by this phenomenon as cancer tissues are
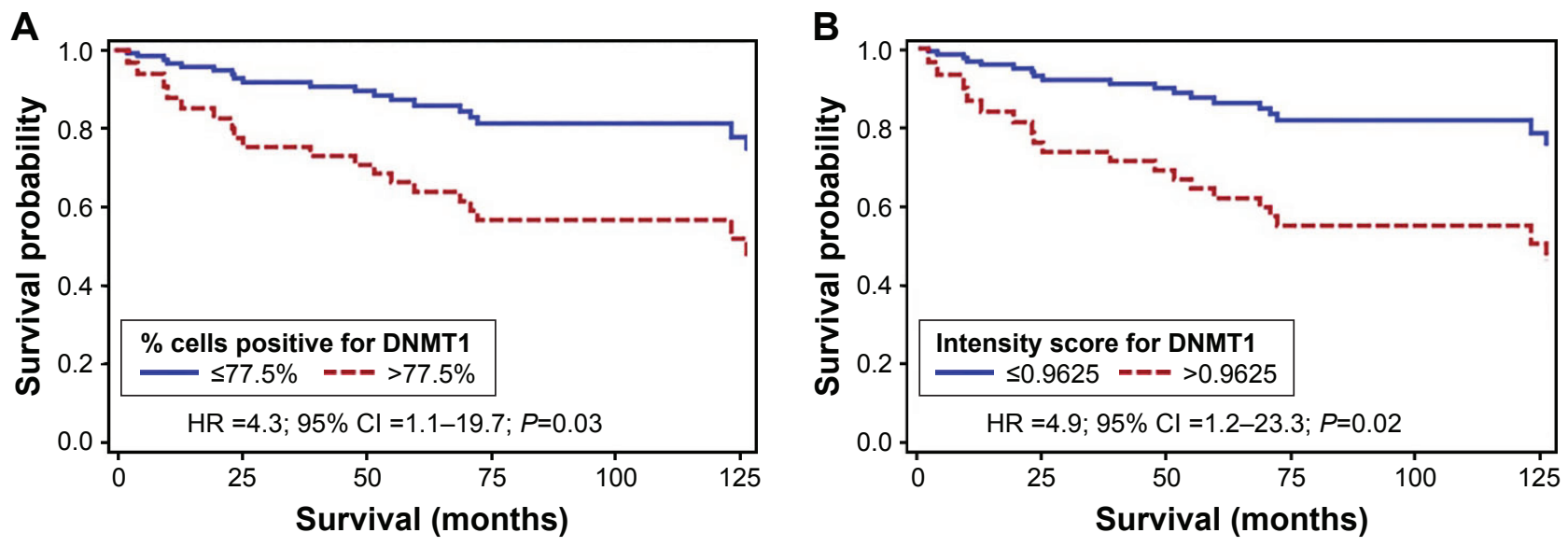

Figure 3 Kaplan-Meier survival curves for the association between (A) percentage of cells positive for DNMTI and (B) intensity score for DNMTI and overall survival. Notes: Adjusted for age, race, stage, histological subtype and mode of treatment.

Abbreviations: DNMT, DNA methyltransferase; $\mathrm{HR}$, hazard ratio; $\mathrm{Cl}$, confidence interval. 
more likely to have HR-HPV infections expressing E6/E7 compared to normal tissues.

Even though the exact nature of the defect in methylation machinery of cancer cells remains unclear, it is thought to be related to the expression of DNMTs, primarily, DNMT1, DNMT3a and DNMT3b. ${ }^{16}$ DNMT catalyzes the transfer of a methyl group from S-adenosylmethionine (AdoMet) to the 5-position of cytosine in DNA, and the specific pattern of methylation provides differential accessibility to the DNA information code by affecting DNA-protein interactions and chromatin condensation, and DNMT1 is best known as the maintenance methyltransferase that copies methylation patterns after DNA replication. ${ }^{17}$ DNMT1 has been shown to recognize and bind with high affinity to DNA lesions such as base mispairs, uracil and other unusual DNA conformations, ${ }^{18,19}$ and these lesions are thought to be present in human cancerous lesions. This phenomenon may also explain the higher expression of DNMT1 in CC lesions compared to normal cervical epithelium. It has been hypothesized that the high-affinity binding of the DNMT1 to un-repaired lesions in DNA could sequester available enzyme away from the replication fork and promote passive replication-dependent demethylation. ${ }^{20}$ It has also been hypothesized that when DNA lesions occur within the promoter region of cancer-promoting genes, the aberrant highaffinity binding of the methyltransferase to these lesions is associated with ectopic cytosine methylation. Therefore, in general, the increased DNMT1 activity is associated with genome-wide hypomethylation and regional hypermethylation in CpG islands of tumor suppressor genes, ${ }^{21}$ and both of these DNA changes are associated with an increased risk of cancer.

Epigenetic therapy, based on the use of epigenetic alterations as therapeutic drugs, is increasingly recognized as a mainstream method for the development of new biomarkers and targets for cancer therapy ${ }^{22,23}$ since DNA methylation is a reversible process in which genes could get demethylated and then restored to their original status. Because of this reversibility of DNA methylation, DNMTis have been investigated as anticancer agents as they are able to restore the expression of genes, for example, tumor suppressor genes, by blocking the activity of DNMTs. ${ }^{24}$ The Food and Drug Administration (FDA)-approved DNMTis include azacitidine and decitabine (5-aza-2'-deoxycytidine) developed in 2004 and 2006, respectively, which have been used for the treatment of myelodysplastic syndrome. ${ }^{25}$ Zebularine is another nucleoside analog developed later that has been shown to result in demethylation and reactivation of a silenced and hypermethylated p16 gene in human bladder tumor cells grown in nude mice. ${ }^{26}$ Novel nucleoside analogs with lower toxicity and better pharmacokinetics and bioavailability (4'-thio-2'-deoxycytidine [T-dCyd] and 5-aza-4'-thio-2'deoxycytidine [aza-T-dCyd]) have the ability to potently deplete DNMT1 in both in vitro and in vivo models of cancer and in human tumor xenografts. ${ }^{27}$ In addition, studies have shown that non-nucleosides such as hydralazine have the ability to demethylate and restore the expression of genes silenced by promoter hypermethylation in primary tumors. ${ }^{28}$ Differential DNA methylation signatures representing several mechanisms in the cellular machinery that are epigenetically deregulated by DNA methylation have been identified in CCs. However, to our knowledge, no studies have documented the expression of DNMT1 in CCs, and as a result, unlike with other cancers, epigenetic therapy tailored based on the expression of this important enzyme for CC is still in its infancy. Preclinical studies, however, have confirmed the DNA methylation inhibitory activity of hydralazine on genes such as $A P C$ in $C C .{ }^{29}$ A Phase I study that focused on the methylation status of $A P C, M G M T, E R, G S T P 1, D A P K$, $R A R \beta$, FHIT and $p 16$ genes showed that hydralazine was effective in demethylating and reactivating the expression of several tumor suppressor genes without affecting global DNA methylation in cervical SCC. ${ }^{30}$ However, aberrant DNA methylation signatures in CCs have varied across different studies, ${ }^{31-33}$ suggesting the importance of identifying other methylation-related markers that are associated with the prognosis of CC. Overexpression of DNMT could potentially be an "alternate marker" to methylation signatures as this enzyme is involved in establishing aberrant DNA methylation patterns in several cancers.

Several studies have evaluated the expression of DNMTs in cancer tissues, particularly, DNMT1, and reported variable levels of overexpression or its association with shorter overall survival of patients treated for lung cancer, ${ }^{34,35}$ pancreatic cancer, ${ }^{36}$ gastric cancer ${ }^{37}$ and gastrointestinal diffuse large B-cell lymphoma. ${ }^{38}$ In these studies, however, it is unclear whether the expression of DNMT1 was evaluated in normal epithelium of the same individuals. Therapy against DNMT1 is more likely to be clinically useful when its expression is higher in cancer cells compared to normal cells of the same individual. In our study, we demonstrated that the expression of DNMT1 is significantly higher in CC cells compared to normal or non-cancer cells, suggesting that DNMTis are likely to be an effective and targeted therapy for CC. Our observation that a higher expression of DNMT1 was significantly associated with poor survival outcome further 
assures that treatment with DNMTis alone or in combination with immunotherapy would be a novel approach for improving survival of women diagnosed with CC.

Even though the oncogenes of HR-HPVs are capable of upregulating DNMT1, other mechanisms may also be involved as this enzyme is regulated by HR-HPV-independent pathways such as adenomatous polyposis coli $(A P C)$ gene in colorectal cancer. ${ }^{39}$ Whether such pathways operate in CC is unknown but likely, and the effects of those on upregulation of DNMT1 along with its upregulation by HPV oncoproteins suggest that DNMT1 should be explored as a target for the treatment of CC. Even though the expression of DNMT1 was higher in SCCs compared to ACs in general, our observation that ACs expressing higher DNMT1 are significantly more likely to have poor survival outcome compared to SCCs expressing higher DNMT1 suggested that treatment with DNMTis could be effective for both AC and SCC depending on the degree of expression of this enzyme. Replication of our results in other study populations that include sufficient numbers of ACs and SCCs is needed prior to considering tailored treatments with these epigenetic therapies. Lack of information, such as body mass index (BMI), dose, and period of treatment, and smoking status could be a limitation of our study. However, lack of such data is unlikely to affect the results in a significant way as one specific group is unlikely to be different by these variables. A detailed evaluation of the expression of DNMT1-paired normal and cancer tissues and documentation of survival outcomes after adjusting for relevant covariates are strengths of the study. Evaluation of only DNMT1 rather than all DNMTs could also be viewed as a limitation of the study, but other studies have documented that DNMT1 is the most discriminatory marker of poor survival outcome compared to other DNMTs in HPV-related oral cancer. ${ }^{40}$

\section{Conclusion}

We document that the expression of DNMT1 is higher in $\mathrm{CC}$ tissues compared to that in normal tissues and its higher expression is significantly associated with poor survival outcomes, suggesting that the degree of expression of this enzyme could be considered as a target in the treatment of CC.

\section{Acknowledgment}

This study was supported by the NCI U54 CA118948 (UAB).

\section{Disclosure}

The authors report no conflicts of interest in this work.

\section{References}

1. Jemal A, Bray F, Center MM, Ferlay J, Ward E, Forman D. Global cancer statistics. CA Cancer J Clin. 2011;61(2):69-90.

2. American Cancer Society [webpage on the Internet]. What Are the Key Statistics about Cervical Cancer? Available from: http://www.cancer. org/cancer/cervicalcancer/detailedguide/cervical-cancer-key-statistics. Accessed June 16, 2016

3. McNeil C. New standard of care for cervical cancer sets stage for next questions. J Natl Cancer Inst. 1999;91(6):500-501.

4. Howlader N, Noone AM, Krapcho M, et al, editors [webpage on the Internet]. SEER Cancer Statistics Review, 1975-2010. Bethesda, MD: National Cancer Institute; 2013. Available from: http://seer.cancer. gov/csr/1975_2010/, based on November 2012 SEER data submission, posted to the SEER web site. Accessed April 3, 2016.

5. Jair KW, Bachman KE, Suzuki H, et al. De novo CpG island methylation in human cancer cells. Cancer Res. 2006;66(2):682-692.

6. Oh BK, Kim H, Park HJ, et al. DNA methyltransferase expression and DNA methylation in human hepatocellular carcinoma and their clinicopathological correlation. Int J Mol Med. 2007;20(1):65-73.

7. Chiappinelli KB, Zahnow CA, Ahuja N, Baylin SB. Combining epigenetic and immunotherapy to combat cancer. Cancer Res. 2016;76(7): $1683-1689$.

8. Piyathilake CJ, Celedonio JE, Macaluso M, Bell WC, Azrad M, Grizzle WE. Mandatory fortification with folic acid in the United States is associated with increased expression of DNA methyltransferase-1 in the cervix. Nutrition. 2008;24(1):94-99.

9. Vaccarella S, Lortet-Tieulent J, Plummer M, Franceschi S, Bray F. Worldwide trends in cervical cancer incidence: impact of screening against changes in disease risk factors. Eur J Cancer. 2013;49(15): 3262-3273.

10. Vizcaino AP, Moreno V, Bosch FX, Muñoz N, Barros-Dios XM, Parkin DM. International trends in the incidence of cervical cancer: I. Adenocarcinoma and adenosquamous cell carcinomas. Int $J$ Cancer. 1998;75(4):536-545.

11. Vizcaino AP, Moreno V, Bosch FX, et al. International trends in incidence of cervical cancer: II. Squamous-cell carcinoma. Int J Cancer. 2000;86(3):429-435.

12. Wang SS, Sherman ME, Hildesheim A, Lacey JV Jr, Devesa S. Cervical adenocarcinoma and squamous cell carcinoma incidence trends among white women and black women in the United States for 1976-2000 Cancer. 2004;100(5):1035-1044.

13. Smith HO, Tiffany MF, Qualls CR, Key CR. The rising incidence of adenocarcinoma relative to squamous cell carcinoma of the uterine cervix in the United States - a 24-year population-based study. Gynecol Oncol. 2000;78(2):97-105.

14. Bray F, Carstensen B, Møller H, et al. Incidence trends of adenocarcinoma of the cervix in 13 European countries. Cancer Epidemiol Biomarkers Prev. 2005;14(9):2191-2199.

15. de Sanjose S, Quint WG, Alemany L, et al; Retrospective International Survey and HPV Time Trends Study Group. Human papillomavirus genotype attribution in invasive cervical cancer: a retrospective crosssectional worldwide study. Lancet Oncol. 2010;11(11):1048-1056.

16. Leonhardt H, Bestor TH. Structure, function and regulation of mammalian DNA methyltransferase. EXS. 1993;64:109-119.

17. Geiman TM, Robertson KD. Chromatin remodeling, histone modifications, and DNA methylation - how does it all fit together? J Cell Biochem. 2002;87(2):117-125.

18. Klimasauskas S, Roberts RJ. Hhal binds tightly to substrates containing mismatches at the target site. Nucleic Acids Res. 1995;23(8):1388-1395.

19. Yang AS, Shen JC, Zingg JM, Mi S, Jones PA. Hha and HpaII DNA methyltransferases bind DNA mismatches, methylate uracil and block DNA repair. Nucleic Acids Res. 1995;23:1380-1386.

20. James SJ, Pogribny IP, Pogribna M, Miller BJ, Jernigan S, Melnyk S. Mechanisms of DNA damage, DNA hypomethylation, and tumor progression in the folate/methyl-deficient rat model of hepatocarcinogenesis. $J$ Nutr. 2003;133(11 suppl 1):3740S-3747S. 
21. Laird PW, Jaenisch R. DNA methylation and cancer. Hum Mol Genet. 1994;3(1):1487-1495.

22. Rodriguez-Paredes M, Esteller M. Cancer epigenetics reaches mainstream oncology. Nat Med. 2011;17(3):330-339.

23. Kelly TK, De Carvalho DD, Jones PA. Epigenetic modifications as therapeutic targets. Nat Biotechnol. 2010;28(10):1069-1078.

24. Szyf M. DNA methylation and demethylation as targets for anticancer therapy. Biochemistry. 2005;70(5):533-549.

25. Derissen EJ, Beijnen JH, Schellens JH. Concise drug review: azacitidine and decitabine. Oncologist. 2013;18(5):619-624.

26. Cheng JC, Matsen CB, Gonzales FA, et al. Inhibition of DNA methylation and reactivation of silenced genes by zebularine. $J$ Natl Cancer Inst. 2003;95(5):399-409.

27. Thottassery JV, Sambandam V, Allan PW, et al. Novel DNA methyltransferase-1 (DNMT1) depleting anticancer nucleosides, 4'-thio-2'-deoxycytidine and 5-aza-4'-thio-2'-deoxycytidine. Cancer Chemother Pharmacol. 2014;74(2):291-302.

28. Singh N, Dueñas-González A, Lyko F, Medina-Franco JL. Molecular modeling and molecular dynamics studies of hydralazine with human DNA methyltransferase 1. ChemMedChem. 2009;4(5):792-799.

29. Song Y, Zhang C. Hydralazine inhibits human cervical cancer cell growth in vitro in association with APC demethylation and reexpression. Cancer Chemother Pharmacol. 2009;63(4):605-613.

30. Zambrano P, Segura-Pacheco B, Perez-Cardenas E, et al. A phase I study of hydralazine to demethylate and reactivate the expression of tumor suppressor genes. BMC Cancer. 2005;5:44.

31. Wei X, Zhang S, Cao D, et al. Aberrant hypermethylation of SALL3 with HPV involvement contributes to the carcinogenesis of cervical cancer. PLoS One. 2015;10(12):e0145700.
32. Yang WT, Zheng PS. Promoter hypermethylation of KLF4 inactivates its tumor suppressor function in cervical carcinogenesis. PLoS One. 2014;9(2):e88827.

33. Guenin S, Mouallif M, Deplus R, et al. Aberrant promoter methylation and expression of UTF1 during cervical carcinogenesis. PLoS One. 2012;7(8):e42704.

34. Kim H, Kwon YM, Kim JS, et al. Elevated mRNA levels of DNA methyltransferase- 1 as an independent prognostic factor in primary non-small cell lung cancer. Cancer. 2006;107(5):1042-1049.

35. Vallböhmer D, Brabender J, Yang D, et al. DNA methyltransferases messenger RNA expression and aberrant methylation of $\mathrm{CpG}$ islands in non-small-cell lung cancer: association and prognostic value. Clin Lung Cancer. 2006;8:39-44.

36. Wang W, Gao J, Man XH, Li ZS, Gong YF. Significance of DNA methyltransferase-1 and histone deacetylase-1 in pancreatic cancer. Oncol Rep. 2009;21(6):1439-1447.

37. Mutze K, Langer R, Schumacher F, et al. DNA methyltransferase 1 as a predictive biomarker and potential therapeutic target for chemotherapy in gastric cancer. Eur J Cancer. 2011;47(12):1817-1825.

38. Zhao H, Zhang LE, Guo S, et al. Overexpression of DNA methyltransferase 1 as a negative independent prognostic factor in primary gastrointestinal diffuse large B-cell lymphoma treated with CHOP-like regimen and rituximab. Oncol Lett. 2015;9(5):2307-2312.

39. Campbell PM, Szyf M. Human DNA methyltransferase gene DNMT1 is regulated by the APC pathway. Carcinogenesis. 2003;24(1):17-24.

40. Supic G, Kozomara R, Zeljic K, Jovic N, Magic Z. Prognostic value of the DNMTs mRNA expression and genetic polymorphisms on the clinical outcome in oral cancer patients. Clin Oral Investig. 2017; 21(1):173-182.
International Journal of Women's Health

\section{Publish your work in this journal}

The International Journal of Women's Health is an international, peerreviewed open-access journal publishing original research, reports, editorials, reviews and commentaries on all aspects of women's healthcare including gynecology, obstetrics, and breast cancer. The manuscript management system is completely online and includes

\section{Dovepress}

a very quick and fair peer-review system, which is all easy to use. Visit http://www.dovepress.com/testimonials.php to read real quotes from published authors. 